\title{
Levels of Transforming Growth Factor-Beta After Immunization of Mice With in vivo prepared Toxoplasma gondii Excretory/Secretory Proteins
}

\author{
Seyed Hossein Abdollahi ${ }^{1}$; Fateme Ayoobi ${ }^{2}$; Hossein Khorramdelazad ${ }^{1}$; Behzad Nasiri \\ Ahmadabadi ${ }^{3}$; Mohammadtaghi Rezayati ${ }^{3}$; Mohammad Kazemi Arababadi ${ }^{3}$; Mohammad \\ Zare-Bidaki ${ }^{3, *}$ \\ ${ }^{1}$ Molecular Medicine Research Center, Rafsanjan University of Medical Sciences, Rafsanjan, IR Iran \\ ${ }_{2}^{2}$ Physiology and Pharmacology Research Center, Rafsanjan University of Medical Sciences, Rafsanjan, IR Iran \\ ${ }^{3}$ Immunology of Infectious Diseases Research Center, Rafsanjan University of Medical Sciences, Rafsanjan, IR Iran \\ *Corresponding author: Mohammad Zare-Bidaki, Immunology of Infectious Diseases Research Center, Rafsanjan University of Medical Sciences, Rafsanjan, IR Iran. Tel: \\ +98-3915234003, Fax: +98-3915225209, E-mail: mzarebidaki@gmail.com
}

Received: January 24, 2014; Revised: April 9, 2014; Accepted: April 15, 2014

\begin{abstract}
Background: Zoonotic parasite Toxoplasma gondii has a high prevalence in human populations. A suitable vaccine for animals can stop the transmission of infection between animal and human.

Objectives: The aim of this study was to evaluate in vivo prepared excretory/secretory antigens (E/SA) as a potential candidate for immunization against the parasite and its effect on the production of transforming growth factor-beta (TGF- $\beta$ ).

Materials and Methods: Toxoplasma gondii tachyzoites were inoculated in the peritoneal cavity of mice and E/SA was harvested and used in animal immunization with and without adjuvant. Serum levels of anti-E/SA antibodies and TGF- $\beta$ were measured in days $0,3,7,14,28$ and 56 after immunization using ELISA technique. The measurements were statistically analyzed.

Results: Our results showed that the serum levels of anti-E/SA immunoglobulins significantly increased in all of the immunized groups. The differences of the serum levels of TGF- $\beta$ between the groups were statistically significant at days 28 and 56 after immunization with E/SA.

Conclusions: Based on our study, in vivo prepared E/SA may be considered as a good candidate for animal immunization.
\end{abstract}

Keywords: Toxoplasma; Immunization; Transforming Growth Factor-beta; Excretory-Secretory Antigen

\section{Background}

Toxoplasmosis is a common worldwide parasitic infection caused by Toxoplasma gondii $(1,2)$. While toxoplasmosis has mild clinical presentations in immuno-competent individuals, in immuno-suppressed adults or congenitally-infected infants the disease is associated with severe pathological complications and even death (3). Toxoplasma gondii can be mainly transmitted to humans through ingesting undercooked warm-blooded livestock meats, containing tissue cysts, as well as foods or water contaminated with cat feces harboring oocysts (4). The transmission can also occur via contaminated milk (5). Therefore, immunization of animals against $T$. gondii may decline the risk of human infections. Previous studies have used whole inactive tachyzoites (6), purified and recombinant T. gondii proteins, and DNA Molecules (7-9) to make an appropriate immunization against $T$. gondii in animals. None of the abovementioned vaccines could lead to a successful immunization against the parasite.

Toxoplasma gondii excretory-secretory antigens (E/SA), comprising more than $90 \%$ of the total parasite circulating antigens, are the primary targets for the host im- mune system $(2,10)$. E/SAs are the most important toxoplasma antigens which form the majority of circulating antigens in the sera from hosts with acute toxoplasmosis. It seems that E/SA plays an important role in inducing appropriate humoral and cellular immune responses against T. gondii. Although E/SAs are proteins identified in tachyzoites, sporozoites and encysted bradyzoite stages, there is evidence that ESAs released by tachyzoites are highly immunogenic compounds which induce both antibody-dependent and cell-mediated protective immunity, whereas E/SA secreted by bradyzoite causes longlasting immunity to the parasite (10-13). Jongert et al., Hafid et al. and Darcy et al. have evaluated an E/SA derived vaccine candidate from an in vitro cell culture of $T$. gondii parasites $(6,13,14)$. They have shown that E/SA can be considered as a good candidate for inducing immunization against $T$. gondii; however, not yielding 100\% protection $(6,13,14)$. Interestingly, Costa-Silva et al. demonstrated that E/SA derived from in vitro cell culture of T. gondii led to high IgG1, IFN- $\gamma$ and TNF- $\alpha$ production (12). E/SA also induces apoptosis in T-regulatory lymphocytes which is

Copyright (C) 2015, Ahvaz Jundishapur University of Medical Sciences. This is an open-access article distributed under the terms of the Creative Commons Attribution-NonCommercial 4.0 International License (http://creativecommons.org/licenses/by-nc/4.0/) which permits copy and redistribute the material just in noncommercial usages, provided the original work is properly cited. 
an important mechanism to elicit a potential immunization against infectious agents (15).

It seems that in vitro production of antigens cannot lead to appropriate glycosylation (16), and hence, the humoral immunity is unable to recognize native E/SA recovered from in vitro cultures. We previously reported that anti-T. gondii IgG antibody can recognize in vivo prepared E/SA (ivE/SA) (2). Therefore, using E/SA prepared from in vivo culture systems may be considered as a useful vaccine candidate for researchers in this field. Additionally, since cytokines are crucial factors that mediate many immune responses $(17,18)$, an appropriate immunization has to induce suitable cytokine levels. Transforming growth factor-beta (TGF- $\beta$ ) is a cytokines which plays key roles in the regulation of immune cell functions (19). This cytokine leads to inhibition of $\mathrm{B}$ and $\mathrm{T}$ lymphocytes proliferation and induces homeostasis (19). TGF- $\beta$ also contributes to tissue remodeling which happens after infections and injuries (20). This cytokine also contributes to development of Th17 and T-regulatory lymphocytes, which play significant roles in activation and suppression of immune responses, respectively, against parasite infections (20). Evaluation of TGF- $\beta$ could be useful to follow up the status of immune responses in experimental animal models and a good immunization should decrease TGF- $\beta$ production at the first days after immunization.

\section{Objectives}

The aim of this study was to evaluate in vivo prepared E/ SA as an immunization candidate and its effects on the production of TGF- $\beta$ as an anti-inflammatory cytokine in animal models.

\section{Materials and Methods}

\subsection{Production of in vivo Prepared Excretory/Secre- tory Antigens}

Five mice were infected with tachyzoites of T. gondii ( $\mathrm{RH}$ strain) by peritoneal cavity inoculation. In day three after the inoculation, peritoneal fluids were taken and centrifuged $(1000 \times \mathrm{g}$ for 15 minutes). Afterwards, the supernatants were filtered and stored at $-20^{\circ} \mathrm{C}$ as ivE/SAs. The ivE/ SA was measured using the Bradford method.

\subsection{Animal Models}

The study was carried out on 8-10-week-old Balb/C female inbred mice, obtained from the Rafsanjan University of Medical Sciences animal house and kept under standard conditions. The mice were randomly divided into eight groups (with 8-10 mice in each group) as follows; group A was designated as control with no treatment; group B received $10 \mu \mathrm{g}$ of ivE/SA; group C received a solution of $10 \mu \mathrm{g}$ ivE/SA and $100 \mu \mathrm{L}$ complete Freund's adjuvant (CFA); group D received $100 \mu \mathrm{L}$ CFA; group E included mice infected with $T$. gondii which received 10 $\mu$ g ivE/SA; group F included mice infected with T. gondii which received a solution of $10 \mu \mathrm{g}$ ivE/SA and $100 \mu \mathrm{L}$ CFA; group $\mathrm{G}$ were infected with $T$. gondii and received $100 \mu \mathrm{L}$ CFA; and finally, group $\mathrm{H}$ received $100 \mathrm{~mL}$ normal saline solution. The mice were immunized with ivE/SA in days 0 , 3, 7, 14, 28 and 56 and blood samples were obtained three days after the immunization from the tail veins of seven mice per each group and their sera were stored at $-20^{\circ} \mathrm{C}$ until used for the specific antibodies and TGF- $\beta$ analysis. Serum samples taken before the immunization at day 0 were utilized as negative controls.

\subsection{Antibody Assay}

Antibody against E/SA was measured using ELISA technique. ivE/SA was used for capturing specific antibodies against T. gondii in ELISA plates. The following protocol was used for antibody detection: after 16 hours incubation with ivE/SA at $4^{\circ} \mathrm{C}$, the plates were washed five times and incubated for two hours with blocking buffer (including BSA) at room temperature, then washed five times. Serum samples including negative and positive controls were added to the plates after two hours of incubation at room temperature and the plates washed five times. Thereafter, horseradish peroxidase (HRP)-conjugated antimouse antibody was added to the plate wells and incubated for two hours at room temperature and washed five times. Finally, the substrates (Tetramethylbenzidine (TMB)and $\mathrm{H}_{2} \mathrm{O}_{2}$ ) were added for color development for 15 minutes and the reaction was stopped with addition of $\mathrm{H}_{2} \mathrm{SO}_{4}(2 \mathrm{~N})$. The cutoff value was calculated using optical density (OD) values of positive and negative controls. A serial dilution for the positive control was also used for efficacy calculation.

\subsection{Cytokine Assay}

TGF- $\beta$ serum levels of the mice were detected using a commercial ELISA kit (eBioscience, Spain) immediately after the blood collection, according to the manufacturer's guidelines. The sensitivity of the kit was $2 \mathrm{pg} / \mathrm{mL}$ and inter- and intra-assay assessments of reliability of the kit were conducted.

\subsection{Statistical Analysis}

The mean differences of the serum levels of TGF- $\beta$ and specific antibodies against $T$. gondii between the groups in a given time after immunization and in a group in different times were respectively analyzed by analysis of variance (ANOVA) and repeated ANOVA tests, using SPSS 20 software. When P value was less than 0.05 , the difference was considered statistically significant.

\section{Results}

Results of the study showed that the serum levels of antiE/SA immunoglobulins increased in all the groups, except for group $\mathrm{H}$, compared to the control group A (Figure 1). 
Figure 1. Serum Levels of Anti-E/SA Immunoglobulins in the Evaluated Groups

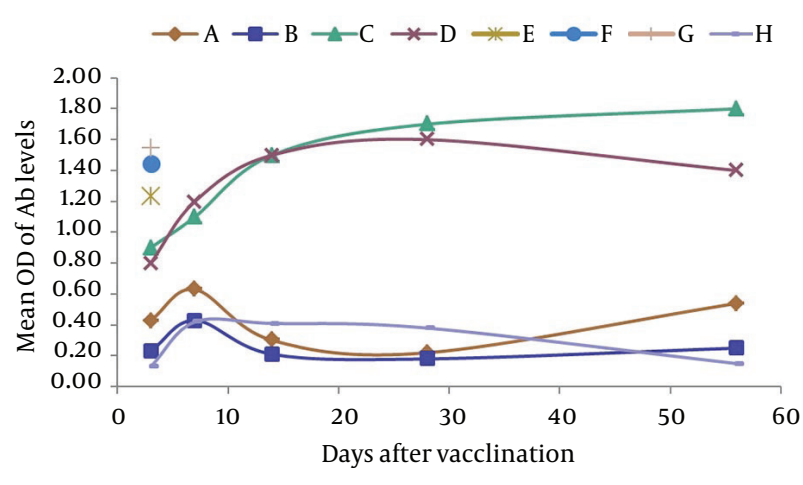

$\mathrm{Y}$ and $\mathrm{X}$ axis display the mean OD of the antibody levels and days after immunizations, respectively.

Figure 2. Serum Levels of TGF- $\beta$ in the Groups

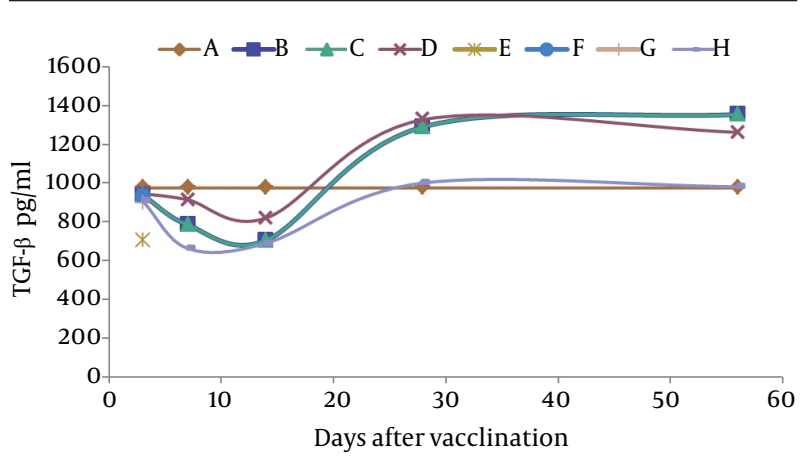

$\mathrm{Y}$ and $\mathrm{X}$ axis display the serum levels of TGF- $\beta$ and the days after immunizations, respectively.

Additionally, all the mice in the groups E, F and G died after five days of inoculation, while the mice in other groups survived. Cytokine assays showed that the differences of the serum levels of TGF- $\beta$ were not statistically significant among the groups at days 3, 7 and 14, but became significant only at days 28 and 56 after immunization with ivE/SA (Figure 2). The results also demonstrated that TGF- $\beta$ serum levels significantly increased in group D only 28 and 56 days after immunization (Figure 2).

\section{Discussion}

Toxoplasmosis is a prevalent parasitic infections, transferred from animals to human (2). Therefore, a good vaccine for stopping the parasite life cycle in animals is essential. Our study showed that in vivo prepared E/SA could induce anti-E/SA antibodies in mice, and thus, it can be considered as a good vaccine candidate for animal immunization. Furthermore, we found that the serum levels of TGF- $\beta$ did not changed in days 3, 7 and 14 after ivE/SA inoculation. Based on the fact that TGF- $\beta$ has anti-inflammatory effects on immune cells as well as on antibody production (19), it seems that immune cells at the early days after immunization are active and respond against E/SA.

Our data regarding anti-E/SA also confirmed this statement. After descripting the elevated levels of TGF- $\beta$ at days 28 and 56 after the immunization, it may be concluded that since the immune system needs to decline immune responses to E/SA and go to homeostasis, TGF- $\beta$ was produced by the immune cells to achieve this goal as a normal immune system response. Previous studies showed that T. gondii induced TGF- $\beta$ secretion by immune cells as an anti-inflammatory agent to decrease the autoimmune responses and the serum levels of the cytokine increased at early phases of infection $(21,22)$. Another investigation also demonstrated that T. gondii induced macrophages apoptosis through autocrine TGF- $\beta$ signaling (23). E/SA also induced apoptosis in T regulatory lymphocytes, which are important cells to produce TGF- $\beta$ (15). Therefore, it may be concluded that ivE/SA inhibited the elevation of TGF- $\beta$ via induction of apoptosis in $\mathrm{T}$ regulatory lymphocytes.

Additionally, our results revealed that both ivE/SA and live $T$. gondii induced a good level of anti-ivE/SA production, but the T. gondii infected mice died after seven days. It appears that during the infection, $T$. gondii proliferates and attacks the parenchyma tissues and antibody production after $T$. gondii proliferation is unable to inhibit the disease. Therefore, in vivo prepared E/SA can be considered as a good candidate for animal immunization, mainly due to the good level of anti-E/SA induction as well as none-increasing TGF-b at the early phases of immunization. Other investigations have used other types of immunization including DNA vaccines $(24,25)$, purified protein (26), and subunit vaccines (27) against $T$. gondii. Similar to our study, antibodies against T. gondii increased in all the studies and cytokine assays showed increase in inflammatory and decrease in the anti-inflammatory cytokines (24-27). For instance, Costa-Silva et al. revealed that E/SA derived from in vitro cell culture of T. gondii elicited high levels of IgG1 and proinflammatory cytokines such as IFN-g and TNF- $\alpha$ (12).

Costa-Silva et al. also reported that E/SA derived from in vitro cell culture of $T$. gondii increased the survival of infected mice (12). Several studies have also evaluated E/SA derived from cell cultures and have reported that these antigens have been useful for eliciting good levels of immune responses in immunized animals (10, 13, 28-30). The preferences of our study may include the followings: a) since E/SA was prepared from an in vivo approach, it may induce anti-E/SA response with higher avidity; b) the preparation of E/SA in mice peritoneal cavity is cheaper and easier than other methods. Interestingly, our previous study also showed that serum levels of IL-10, an antiinflammatory cytokine, did not change at the early phases of immunization with ivE/SA (11).

Finally, T. gondii has several mechanisms to escape from the host immune responses and it is necessary to evalu- 
ate more aspects of immunization such as cellular immunity and animal survival.

According to our results, ivE/SA can be considered as a good candidate for immunization against $T$. gondii and further studies on human can elucidate the immunogenicity of ivE/SA in comparison with E/SA derived from in vitro cell culture of $T$. gondii.

\section{Acknowledgements}

The authors would like to take this opportunity to thank all the staff of the Molecular and Medicine Research Center as well as Rafsanjan University of Medical Sciences Animal House, who warmly helped us with conducting this research project.

\section{Authors' Contributions}

Seyed Hossein Abdollahi, Mohammad Kazemi Arababadi and Mohammad Zare-Bidaki: study design and supervision, literature review and manuscript writing and revision. Fateme Ayoobi, Hossein Khorramdelazad, Behzad Nasiri Ahmadabadi and Mohammadtaghi Rezayati: data collection, cytokine and immunoglobulins assays, animal practice and manuscript writing and review.

\section{Financial Disclosure}

The authors had no financial interests related to the materials in the manuscript.

\section{Funding/Support}

Rafsanjan University of Medical Sciences financially supported the project.

\section{References}

1. Rinaldi L, Scala A. Toxoplasmosis in livestock in Italy: an epidemiological update. Parassitologia. 2008;50(1-2):59-61.

2. Abdollahi SO, Arababadi MK, Hassanshahi G. Evaluation of excreted/secreted antigens derived from peritoneal of toxoplasma infected small mice to detect IgG against Toxoplasma. PakJ Biol Sci. 2009;12(6):530-3.

3. Nissapatorn V. Toxoplasmosis in HIV|AIDS: a living legacy. Southeast Asian J Trop Med Public Health. 2009;40(6):1158-78.

4. Hide G, Morley EK, Hughes JM, Gerwash O, Elmahaishi MS, Elmahaishi $\mathrm{KH}$, et al. Evidence for high levels of vertical transmission in Toxoplasma gondii. Parasitology. 2009;136(14):1877-85

5. Blokzijl ML. Human immunodeficiency virus infection in childhood. Ann Trop Paediatr. 1988;8(1):1-17.

6. Jongert E, Roberts CW, Gargano N, Forster-Waldl E, Petersen E. Vaccines against Toxoplasma gondii: challenges and opportunities. Mem Inst Oswaldo Cruz. 2009;104(2):252-66.

7. Angus CW, Klivington-Evans D, Dubey JP, Kovacs JA. Immunization with a DNA plasmid encoding the SAG1 (P30) protein of Toxoplasma gondii is immunogenic and protective in rodents. J Infect Dis. 2000;181(1):317-24.

8. El-Malky M, Shaohong L, Kumagai T, Yabu Y, Noureldin MS, Saudy $\mathrm{N}$, et al. Protective effect of vaccination with Toxoplasma lysate antigen and $\mathrm{CPG}$ as an adjuvant against Toxoplasma gondii in susceptible C57BL/6 mice. Microbiol Immunol. 2005;49(7):639-46.

9. Liu KY, Zhang DB, Wei QK, Li J, Li GP, Yu JZ. Biological role of surface Toxoplasma gondii antigen in development of vaccine. World J Gastroenterol. 2006;12(15):2363-8.

10. Daryani A, Hosseini AZ, Dalimi A. Immune responses against excreted/secreted antigens of Toxoplasma gondii tachyzoites in the murine model. Vet Parasitol. 2003;113(2):123-34.

11. Abdollahi SH, Ayoobi F, Khorramdelazad H, Hassanshahi G, Ahmadabadi BN, Rezayati M, et al. Interleukin-10 Serum Levels after Vaccination with In Vivo Prepared Toxoplasma gondii Excreted/ Secreted Antigens. Oman Med J. 2013;28(2):112-5.

12. Costa-Silva TA, Borges MM, Galhardo CS, Pereira-Chioccola VL. Immunization with excreted/secreted proteins in AS/n mice activating cellular and humoral response against Toxoplasma gondii infection. Acta Trop. 2012;124(3):203-9.

13. Hafid J, Vincent N, Flori P, Bellete B, Raberin H, Sung RT. Production of antibodies in murine mucosal immunization with Toxoplasma gondii excreted/secreted antigens. Vet Parasitol. 2005;128(1-2):23-8.

14. Darcy F, Deslee D, Santoro F, Charif H, Auriault C, Decoster A, et al. Induction of a protective antibody-dependent response against toxoplasmosis by in vitro excreted/secreted antigens from tachyzoites of Toxoplasma gondii. Parasite Immunol. 1988;10(5):553-67.

15. Chen JL, Ge YY, Zhang J, Qiu XY, Qiu JF, Wu JP, et al. The dysfunction of $\mathrm{CD} 4(+) \mathrm{CD} 25(+)$ regulatory $\mathrm{T}$ cells contributes to the abortion of mice caused by Toxoplasma gondii excreted-secreted antigens in early pregnancy. PLoS One. 2013;8(7):e69012.

16. Odenthal-Schnittler M, Tomavo S, Becker D, Dubremetz JF, Schwarz RT. Evidence for N-linked glycosylation in Toxoplasma gondii. Biochem J.1993;291:713-21.

17. Arababadi MK, Mosavi R, Khorramdelazad H, Yaghini N, Zarandi ER, Araste M, et al. Cytokine patterns after therapy with Avonex(R), Rebif(R), Betaferon(R) and CinnoVex in relapsingremitting multiple sclerosis in Iranian patients. Biomark Med. 2010;4(5):755-9.

18. Ahmadabadi BN, Hassanshahi G, Arababadi MK, Leanza C, Kennedy D. The IL-10 promoter polymorphism at position -592 is correlated with susceptibility to occult HBV infection. Inflammation. 2012;35(3):818-21.

19. Kehrl JH. Transforming growth factor-beta: an important mediator of immunoregulation. Int J Cell Cloning. 1991;9(5):438-50.

20. Karimi-Googheri M, Daneshvar H, Nosratabadi R, Zare-Bidaki M Hassanshahi G, Ebrahim M, et al. Important roles played by TGFbeta in hepatitis B infection. J Med Virol. 2014;86(1):102-8.

21. Wagner A, Forster-Waldl E, Garner-Spitzer E, Schabussova I, Kundi $\mathrm{M}$, Pollak A, et al. Immunoregulation by Toxoplasma gondii infection prevents allergic immune responses in mice. Int J Parasitol. 2009;39(4):465-72.

22. Kasper L, Courret N, Darche S, Luangsay S, Mennechet F, Minns L, et al. Toxoplasma gondii and mucosal immunity. Int J Parasitol. 2004;34(3):401-9.

23. D'Angelillo A, De Luna E, Romano S, Bisogni R, Buffolano W, Gargano N, et al. Toxoplasma gondii Dense Granule Antigen 1 stimulates apoptosis of monocytes through autocrine TGF-beta signaling. Apoptosis. 2011;16(6):551-62.

24. Makino M, Uemura N, Moroda M, Kikumura A, Piao LX, Mohamed $\mathrm{RM}$, et al. Innate immunity in DNA vaccine with Toxoplasma gondii-heat shock protein 70 gene that induces DC activation and Th1 polarization. Vaccine. 2011;29(10):1899-905.

25. Yan HK, Yuan ZG, Petersen E, Zhang XX, Zhou DH, Liu Q, et al Toxoplasma gondii: protective immunity against experimental toxoplasmosis induced by a DNA vaccine encoding the perforinlike protein 1. Exp Parasitol. 2011;128(1):38-43.

26. Cong H, Mui EJ, Witola WH, Sidney J, Alexander J, Sette A, et al Towards an immunosense vaccine to prevent toxoplasmosis: protective Toxoplasma gondii epitopes restricted by HLA-A*0201. Vaccine. 2011;29(4):754-62.

27. Dziadek B, Gatkowska J, Brzostek A, Dziadek J, Dzitko K, Grzybowski $\mathrm{M}$, et al. Evaluation of three recombinant multi-antigenic vaccines composed of surface and secretory antigens of Toxoplasma gondii in murine models of experimental toxoplasmosis. Vaccine. 2011;29(4):821-30.

28. Prigione I, Facchetti P, Lecordier L, Deslee D, Chiesa S, CesbronDelauw MF, et al. T cell clones raised from chronically infected healthy humans by stimulation with Toxoplasma gondii excretory-secretory antigens cross-react with live tachyzoites: 


\section{Abdollahi SH et al.}

characterization of the fine antigenic specificity of the clones and implications for vaccine development. J Immunol. 2000;164(7):3741-8.

29. Quan JH, Zhou W, Cha GH, Choi IW, Shin DW, Lee YH. Kinetics of IL23 and IL-12 secretion in response to Toxoplasma gondii antigens from THP-1 monocytic cells. Korean J Parasitol. 2013;51(1):85-92.

30. Rahmah N, Anuar A. Comparison of three forms of antigens in the demonstration of cell-mediated immune response in $\mathrm{mu}-$ rine toxoplasmosis. Biochemical and Biophysical Research Communications. 1992;189(2):640-4 